\title{
An Exactly Solvable Model for the Fermi Contact Interaction
}

\author{
S. M. Blinder \\ Department of Chemistry, University of Michigan, Ann Arbor, Michigan 48109, USA
}

\begin{abstract}
A model for the Fermi contact interaction is proposed in which the nuclear moment is represented as a magnetized spherical shell of radius $r_{0}$. For a hydrogen-like system thus perturbed, the Schrödinger equation is solvable without perturbation theory by use of the Coulomb Green's function. Approximation formulas are derived in terms of a quantum defect in the Coulombic energy formula. It is shown that the usual Fermi potential cannot be applied beyond first-order perturbation theory.
\end{abstract}

Key words: Fermi contact interaction

Atomic hyperfine interactions involving $s$-electrons are well accounted for by the Fermi contact operator $[1,2]$

$$
\begin{aligned}
\mathscr{H}^{\prime} & =\lambda \pi a^{3} \boldsymbol{s} \cdot I \delta^{3}(\boldsymbol{r}) \\
\lambda & \equiv \frac{8}{3} g g_{I} \mu_{B} \mu_{N} / a^{3}, \quad \delta^{3}(\boldsymbol{r})=\delta(r) / 4 \pi r^{2} .
\end{aligned}
$$

Additionally, nuclear spin-spin coupling in NMR can be attributed predominantly to a second-order mechanism involving the Fermi contact interaction [3]. An unfortunate concomitant to this mechanism is the appearance of divergent nuclear magnetic self-interactions [4]. These divergences are unphysical. The problem shows up in fact even when (1) is applied to the second-order perturbation energy of a hydrogen atom [5]. An extensive literature exists on attempts to circumvent this difficulty in calculations of spin-spin coupling constants in molecules, notably HD [6].

We should like to propose as an alternative, a model for the contact interaction which leads to an exactly solvable atomic problem. Thereby, spin-spin coupling can be treated as a first-order perturbation and divergences are avoided entirely. In 
physical terms, the point nuclear magnetic dipole implied by the Fermi Hamiltonian (1) is to be replaced by a uniformly magnetized spherical shell of radius $r_{0}$. This is effected simply by the substitution in Eq. (1):

$$
\delta(r) \rightarrow \delta\left(r-r_{0}\right) \text {. }
$$

We designate the resultant operator as the modified Fermi potential [7].

A hydrogen-like system perturbed by a modified Fermi potential is represented by the Schrödinger equation (for $s$-states):

$$
\left\{-\frac{\hbar^{2}}{2 \mu} \nabla^{2}-\frac{Z e^{2}}{r}+\frac{8 \pi}{3} g g_{I} \mu_{B} \mu_{N} s \cdot I \frac{\delta\left(r-r_{0}\right)}{4 \pi r_{0}^{2}}\right\} \psi(r)=E \psi(r)
$$

We adopt the modified atomic units

$$
\hbar=c=\mu=1, \quad \mu=m(1+m / M)^{-1}, \quad a \equiv \hbar^{2} / \mu e^{2}=1
$$

and introduce the radial function $P(r)$ such that

$$
\psi(r)=P(r) /\left(4 \pi r^{2}\right)^{1 / 2}
$$

and the wavenumber $k$ where

$$
E=k^{2} / 2 \text {. }
$$

The Schrödinger equation then reduces to the form

$$
\left(k^{2}+\frac{\partial^{2}}{\partial r^{2}}+\frac{2 Z}{r}\right) P(r)=\frac{\lambda_{F}}{2 r_{0}^{2}} P\left(r_{0}\right) \delta\left(r-r_{0}\right)
$$

where

$$
\lambda_{F} \equiv(s \cdot I)_{F} \lambda, \quad(s \cdot I)_{F}=\frac{1}{2}[F(F+1)-s(s+1)-I(I+1)], \quad F=I \pm \frac{1}{2} .
$$

For atomic hydrogen $\left(Z=1, I=\frac{1}{2}\right)$, using the experimental free-electron $g$-factor $g=2.0023193134$

$$
\lambda=2.1600 \times 10^{-7}, \quad \lambda_{1}=\frac{1}{4} \lambda, \quad \lambda_{0}=-\frac{3}{4} \lambda .
$$

Now Eq. (7) is isomorphous with the defining equation for the $S$-wave Coulomb Green's function [8]

$$
\left(k^{2}+\frac{\partial^{2}}{\partial r^{2}}+\frac{2 Z}{r}\right) g\left(r, r_{0}, k\right)=\delta\left(r-r_{0}\right)
$$

having defined

$$
g\left(r, r_{0}, k\right) \equiv r r_{0} G_{0}\left(r, r_{0}, k\right) .
$$

The appropriate boundary conditions are

$$
r^{-1 / 2} g\left(r, r_{0}, k\right) \rightarrow 0, \quad r^{1 / 2} \partial g / \partial r \rightarrow 0 \quad \text { as } r \rightarrow 0
$$

and

$$
g\left(r, r_{0}, k\right) \rightarrow 0, \quad \partial g / \partial r \rightarrow 0 \quad \text { as } r \rightarrow \infty
$$


which are applicable as well to the Schrödinger equation (7). Thus the latter must possess solutions of the form

$$
P_{v}(r)=\frac{\lambda_{F}}{2 r_{0}^{2}} P_{v}\left(r_{0}\right) g\left(r, r_{0}, k\right)
$$

provided that $k$ does not lie in the unperturbed Coulomb eigenvalue spectrum. The Green's function is given by [8]

$$
g\left(r, r_{0}, k\right)=(2 i k)^{-1} \Gamma(1-i v) M_{i v}^{1 / 2}\left(-2 i k r_{<}\right) W_{i v}^{1 / 2}\left(-2 i k r_{>}\right)
$$

where $\nu \equiv Z / k, \operatorname{Im} k>0, M$ and $W$ are Whittaker functions as defined by Buchholz [9]. Since we shall be concerned with bound states, it is expedient to make the substitutions

$$
i \nu \rightarrow \nu, \quad-i k \rightarrow k .
$$

Accordingly

$$
g\left(r, r_{0}, k\right)=-(2 k)^{-1} \Gamma(1-\nu) M_{\nu}\left(2 k r_{<}\right) W_{v}\left(2 k r_{>}\right) .
$$

We have in addition dropped the second index on the Whittaker functions since it will have the value $\frac{1}{2}$ throughout. In place of (6) we have now

$$
E_{v}=-k^{2} / 2=-Z^{2} / 2 \nu^{2}
$$

which has the same form as for Coulomb eigenvalues but with non-integral values of the quantum number $\nu$. For $\lambda \ll 1\left(\lambda \sim 10^{-7}\right.$ for hydrogen) it can be anticipated that the bound state energies given by (16) will differ only minutely from the values $E_{n}=-Z^{2} / 2 n^{2}$. It is convenient to introduce a quantum defect such that

$$
\nu=n+\delta, \quad n=1,2, \ldots
$$

Then

$$
E_{v}=-\frac{Z^{2}}{2 n^{2}}+\frac{Z^{2}}{n^{3}} \delta+O\left(\delta^{2}\right) .
$$

A consistency condition on the eigenfunctions (13), obtained by setting $r=r_{0}$, gives a transcendental equation determining the bound state eigenvalues, viz.,

$$
\frac{\lambda_{F}}{2 r_{0}^{2}} g\left(r_{0}, r_{0}, k\right)=1 \text {. }
$$

Now the $S$-wave Coulomb Green's function defined by (9) has the spectral representation

$$
g\left(r, r_{0}, \varepsilon\right)=S_{n} \frac{P_{n}(r) P_{n}\left(r_{0}\right)}{\varepsilon-\varepsilon_{n}}, \quad \varepsilon \equiv-Z^{2} / \nu^{2}
$$

in terms of the unperturbed $s$-state radial functions $P_{n}(r)$. In the discrete spectrum,

$$
P_{n}(r)=(Z / n)^{1 / 2} M_{n}(2 Z r / n), \quad \varepsilon_{n}=-Z^{2} / n^{2} .
$$


For the perturbed state $n=N, \nu=N+\delta$, the Green's function expanded in powers of the quantum defect takes the form

$$
g\left(r, r_{0}, \varepsilon\right)=\left(\frac{N^{3}}{2 Z^{2} \delta}+\frac{3 N^{2}}{4 Z^{2}}\right) P_{N}(r) P_{N}\left(r_{0}\right)+k\left(r, r_{0}, \varepsilon_{N}\right)+O(\delta)
$$

where $k\left(r, r_{0}, \varepsilon_{N}\right)$ is the reduced Coulomb Green's function $[10,11]$

$$
k\left(r, r_{0}, \varepsilon_{N}\right) \equiv \int_{n \neq N} \frac{P_{n}(r) P_{n}\left(r_{0}\right)}{\varepsilon_{N}-\varepsilon_{n}}
$$

Putting (22) into (19), with neglect of contributions $O(\delta)$, and solving for the quantum defect, we obtain

$$
\delta_{N, F} \approx \frac{\frac{\lambda_{F} N^{3}}{4 Z^{2} r_{0}^{2}}\left|P_{N}\left(r_{0}\right)\right|^{2}}{1-\frac{\lambda_{F}}{2 r_{0}^{2}}\left[\frac{3 N^{2}}{4 Z^{2}}\left|P_{N}\left(r_{0}\right)\right|^{2}+k\left(r_{0}, r_{0}, \varepsilon_{N}\right)\right]}
$$

Explicitly for the $1 s$ state, $N=1$,

$$
P_{1}(r)=2 Z^{3 / 2} r e^{-Z r}
$$

and from formulas given by Hameka [10] and Hostler [11]

$$
k\left(r_{0}, r_{0}, \varepsilon_{1}\right)=2 Z r_{0}^{2} e^{-2 Z r_{0}}\left[-\frac{1}{2 Z r_{0}}+\ln \left(2 Z r_{0}\right)-2 Z r_{0}-f\left(2 Z r_{0}\right)+\gamma-\frac{5}{2}\right]
$$

where

$$
\begin{aligned}
f(\rho) & \equiv \int_{0}^{\rho} \frac{e^{x}-1-x}{x^{2}} d x \\
& =-\left(\frac{e^{\rho}-1-\rho}{\rho^{2}}\right)+\operatorname{Ei}(\rho)-\ln \rho-\gamma
\end{aligned}
$$

The $1 s$ quantum defect is thus given by

$$
\delta_{1, F} \approx \frac{\lambda_{F} Z e^{-2 Z r_{0}}}{1+\lambda_{F} Z e^{-2 Z r_{0}}\left[\frac{1}{2 Z r_{0}}-\ln \left(2 Z r_{0}\right)+2 Z r_{0}+f\left(2 Z r_{0}\right)-\gamma+1\right]} .
$$

Under the further approximation that $r_{0} \ll 1$ (bohr)

$$
\delta_{1, F} \approx \frac{\lambda_{F} Z\left(1-2 Z r_{0}\right)}{1+\lambda_{F} / 2 r_{0}} \text {. }
$$

In the more detailed derivation given elsewhere [7], it was shown that Eq. (29) applies more generally to all bound hydrogen-like $s$-states.

For $\lambda_{F}>0$, say the $F=1$ state of hydrogen, the quantum defect (29) converges uniformly to zero as $r_{0} \rightarrow 0$. Thus for a repulsive deltafunction potential, the energy reverts to its unperturbed Coulomb value. For $\lambda_{F}<0$, say the $F=0$ state of hydrogen, Eq. (29) remains valid so long as $\left|\lambda_{F}\right| / 2 r_{0} \ll 1$. The quantum defect 
goes through "resonance" near $r_{0}=\left|\lambda_{F}\right| / 2$. The condition $|\delta| \ll I$ is violated so that the entire derivation becomes invalid. The desired limit can however be obtained by considering the asymptotic forms of Eqs. (15) and (19) as $k \rightarrow \infty$ (or $\nu \rightarrow 0$ ). From formulas given in Buchholz [9]

$$
\Gamma(1-\nu) M_{v}\left(2 k r_{0}\right) W_{y}\left(2 k r_{0}\right) \rightarrow 1 \text { as } k \rightarrow \infty .
$$

Thus

$$
-\frac{\lambda_{F}}{4 k r_{0}^{2}} \approx 1
$$

or

$$
k \approx\left|\lambda_{F}\right| / 4 r_{0}^{2} .
$$

The limit $k \rightarrow \infty$ does indeed correspond to $r_{0} \rightarrow 0$ for negative $\lambda_{F}$. By virtue of (16), $E_{v} \rightarrow-\infty$ as $r_{0} \rightarrow 0$, showing that all bound states are pulled down to $-\infty$ by an attractive Fermi potential. Velenik et al. [12], using variational arguments, arrived at the same conclusions with regard to repulsive and attractive deltafunction potentials added to a Coulombic system.

By expanding the quantum defect (29) in powers of $\lambda_{F}$ and substituting into (18), one obtains a perturbation expansion of the hyperfine interaction energy. As shown in Ref. [7], in the limit $r_{0} \rightarrow 0$, the first-order term approaches the Fermi formula

$$
E^{(1)}=\lambda_{F}\left|\psi_{n}(0)\right|^{2}
$$

while all higher-order perturbation contributions diverge.

\section{References}

1. Fermi, E.: Z. Phys. 60, 320 (1930)

2. Blinder, S. M.: Advan. Quantum Chem. 2, 47 (1965)

3. Ramsey, N. F.: Phys. Rev. 91, 303 (1953)

4. Das, T. P., Bersohn, R.: Phys. Rev. 115, 897 (1959)

5. Schwartz, C.: Ann. Phys. (N.Y.) 2, 156 (1959)

6. Wrubel, H., Voitländer, J.: Mol. Phys. 25, 323 (1973)

7. Blinder, S. M.: Phys. Rev. A18, 853 (1978)

8. Hostler, L.: J. Math. Phys. 5, 591 (1964)

9. Buchholz, H.: The confluent hypergeometric function. New York: Springer, 1969

10. Hameka, H. F.: J. Chem. Phys. 47, 2728 (1967); 48, 4810 (E) (1968)

11. Hostler, L.: Phys. Rev. 178, 126 (1969); J. Math. Phys. 16, 1585 (1975)

12. Velenik, A., Živković, T., de Jeu, W. H., Murrell, J. N.: Mol. Phys. 18, 693 (1970)

Received May 3, 1979 